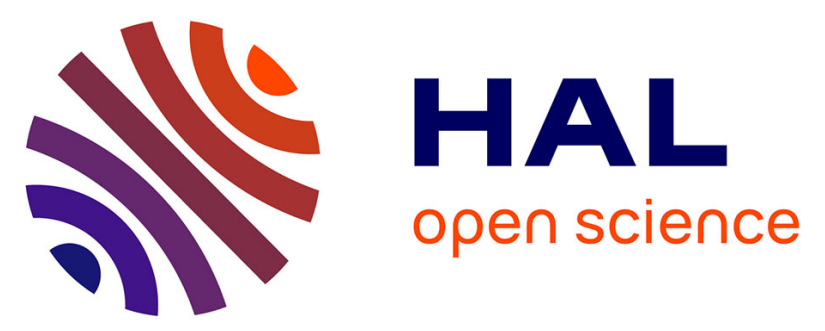

\title{
Continuous and Discrete State Estimation for Switched LPV Systems using Parameter Identification
} Hector Ríos, D Mincarelli, Denis Efimov, Wilfrid Perruquetti, J Davila

\section{To cite this version:}

Hector Ríos, D Mincarelli, Denis Efimov, Wilfrid Perruquetti, J Davila. Continuous and Discrete State Estimation for Switched LPV Systems using Parameter Identification. Automatica, 2015, 62, pp.1-8. 10.1016/j.automatica.2015.09.016 . hal-01188367

\section{HAL Id: hal-01188367 \\ https://inria.hal.science/hal-01188367}

Submitted on 29 Aug 2015

HAL is a multi-disciplinary open access archive for the deposit and dissemination of scientific research documents, whether they are published or not. The documents may come from teaching and research institutions in France or abroad, or from public or private research centers.
L'archive ouverte pluridisciplinaire HAL, est destinée au dépôt et à la diffusion de documents scientifiques de niveau recherche, publiés ou non, émanant des établissements d'enseignement et de recherche français ou étrangers, des laboratoires publics ou privés. 


\title{
Continuous and Discrete State Estimation for Switched LPV Systems using Parameter Identification *
}

\author{
H. Ríos ${ }^{\mathrm{a}}$, D. Mincarelli ${ }^{\mathrm{a}}$, D. Efimov ${ }^{\mathrm{a}, \mathrm{b}}$, W. Perruquetti ${ }^{\mathrm{a}}$ and J. Davila ${ }^{\mathrm{c}}$ \\ ${ }^{\mathrm{a}}$ Non-A team @ Inria, Parc Scientifique de la Haute Borne, 40 avenue Halley, 59650 Villeneuve d'Ascq, France and CRIStAL \\ (UMR-CNRS 9189), Ecole Centrale de Lille, BP 48, Cité Scientifique, 59651 Villeneuve-d'Ascq, France (e-mails: \\ hector.rios_barajas@inria.fr,diego.mincarelli@inria.fr,denis.efimov@inria.fr,wilfrid.perruquetti@inria.fr) \\ ${ }^{\mathrm{b}}$ Department of Control Systems and Informatics, Saint Petersburg State University of Information Technologies Mechanics \\ and Optics (ITMO), 49 Kronverkskiy av., 197101 Saint Petersburg, Russia. \\ ${ }^{\mathrm{c}}$ National Polytechnic Institute, Section of Graduate Studies and Research, ESIME-UPT, C.P. 07340, Mexico D.F., (e-mail: \\ jadavila@ipn.mx)
}

\begin{abstract}
In this paper the problem of discrete and continuous state estimation for a class of uncertain switched LPV systems is addressed. Parameter identification techniques are applied to realize an approximate identification of the scheduled parameters of a switched LPV system with certain uncertainties and/or disturbances. A discrete state estimation is achieved using the parameter identification. A Luenberger-like hybrid observer, based on discrete state information and LMIs approach, is used for the continuous state estimation. The simplicity of the proposed method is one of the main advantages of this paper. The feasibility of the proposed method is illustrated by simulations.
\end{abstract}

Key words: Switched systems; LPV Systems; State estimation.

\section{INTRODUCTION}

In the last two decades, the linear parameter varying (LPV) systems have received a lot of the attention from the control area [17]. Such a kind of systems was introduced by [25] in order to establish a difference between linear time invariant and linear time varying systems. LPV systems represent a class of linear systems whose state/input matrices depend on a set of time varying parameters which can be measured in real time. For processes with mild nonlinearities or dependence on external variables, it has been shown that LPV equivalent representation offers an attractive modeling framework. The practical use of LPV systems is stimulated by the fact that control design for LPV systems is well known (see, e.g. gain scheduling [23], $\mu$-synthesis [30], and linear matrix inequalities (LMIs) based optimal control [24]) since these systems allow us to apply linear design tools to complex nonlinear models. Closely related to the LPV systems, the switched systems are described by the interaction of continuous and discrete state dynam-

\footnotetext{
* This work has been partially presented at 2014 American Control Conference [22]. Corresponding author H. Ríos.
}

ics. This kind of systems has been widely studied during the last decades since they can be used to describe a wide range of physical and engineering systems (see, e.g. [11]). However, for an LPV system with a large parameter variation region, a single controller may not exist or the numerical routines for solution calculation may fail to converge. If such a controller exist, the performance may be sacrificed, in some parameter regions, trying to design a single controller that is able to act over the whole region. A reasonable approach to avoid this kind of problems is to design several LPV systems and controllers, each of them suitable for a specific parameter subregion, and changing among them to fulfill certain possible performance. Then, such a model belongs to a new kind of systems, switched LPV systems. In this context, the switched LPV systems have attracted the attention of many researchers and many results have been achieved about multi-Lyapunov functions for analysis, controller design, and modelling for such systems (see, e.g. [6], [15], [28] where the asynchronous switching estimation problem has been proposed for the first time and the used multiple Lyapunov-like functions approach is allowed to be locally increasable upon which the asynchronous switched filter/observer can be designed, and 
[29]). On the control design, the switched LPV control techniques allow us to use different controllers for each parameter subregion and switch among them according to the evolution of the discrete state. This technique is also beneficial in order to improve the control performance and enhance the design flexibility (see, e.g. [7] and [16]). Nevertheless, most of the switched LPV control approaches are designed taking into account that the discrete state (switching law) is independent of the system parameters, i.e. it is known. For this reason, the knowledge of the discrete state becomes crucial. For switched systems, the discrete state estimation problem has been dealt with in many works. Usually, an observer scheme is designed to estimate the discrete state and then, based on this information, the continuous one can be reconstructed (see, e.g. based on sliding mode observer approach [1], [4], and on algebraic approach [3]). The reverse procedure can also be applied, i.e. discrete state estimation based on the continuous state information, as it is described by [19] and [21], based on sliding mode observers. In [27] necessary and sufficient conditions for a linear switched systems to be invertible are proposed, i.e. condition for recovering the switching signal (discrete state) and the input uniquely. However, to the best of our knowledge, the discrete and continuous state estimation problem in switched LPV systems has not been fully tackled yet. Motivated in the previous explanations and by some recent practical applications (active magnetic bearing systems [15], missile autopilot systems [12], F-16 aircraft system [16], wind turbine [10], and air path system of diesel engine [13]), in this paper the problem of discrete and continuous state estimation for switched LPV systems is addressed. In such systems the discrete state is unknown, and the system behavior can be represented by the interaction of LPV subsystems with unmeasured vector of time-varying scheduling parameters (continuous dynamics) and some commutation operating modes (discrete dynamics).

Main Contribution: A solution to the problem of discrete and continuous state estimation for a class of switched LPV systems under uncertainties is proposed by means of parameter identification techniques and a Luenberger-like hybrid observer, respectively. Taking into account that the time-varying vector of scheduling parameters is unmeasurable, the basic idea to fulfill the aforementioned goal is as follows: 1) The discrete state estimation is achieved by means of the identification of the system parameters (assuming that the parameters lie on different known regions of the parameter space). 2) The continuous state is estimated by means of a hybrid observer that uses the discrete state information.

For the parameter identification, a simple Least Square Method is proposed. Due to one of the objectives is to obtain a practical solution, a simple algorithm is applied. However, the simplicity of the proposed method is one of the main advantages of this work. For the hybrid observer, a Luenberger-like approach is used based on LMIs. Simulation results illustrate the feasibility of the proposed methods.

Structure of the Paper: Section 2 deals with the problem statement and some preliminaries. In section 3 the parameter identification method is described and the algorithm to estimate the discrete state is given. Section 4 shows how to design an observer to estimate the continuous state. The simulation results are shown in section 5 . Finally, some concluding remarks are given in section 6 .

Notation: For a vector $x \in \mathbb{R}^{n}$, the symbol $\|\cdot\|_{\infty}$ denotes the infinity norm, i.e. $\|x\|_{\infty}=\max \left(\left|x_{1}\right|, \ldots,\left|x_{n}\right|\right)$. The Euclidean norm is denoted by $\|\cdot\|_{2}$. Let $\mathbb{R}^{+}=$ $\{t \in \mathbb{R}: t \geq 0\}$. For a locally essentially bounded input $u: \mathbb{R}^{+} \rightarrow \mathbb{R}$ the symbol $\|u\|_{\left[t_{0}, t_{1}\right]}$ denotes its $\mathcal{L}_{\infty}$ norm, i.e. $\|u\|_{\left[t_{0}, t_{1}\right]}=e s s \sup _{t \in\left[t_{0}, t_{1}\right]}\|u(t)\|_{2}$, if $t_{1}=+\infty$ then the symbol $\|u\|$ will be used. Denote by $\mathcal{L}_{\infty}$ the set of all inputs $u$ that satisfy $\|u\|<+\infty$. The convolution operation $*$, on two functions $f$ and $g$, is defined as $f(t) * g(t)=\int_{0}^{t} f(\tau) g(t-\tau) d \tau$. The 1-norm for a function $f:[a, b] \rightarrow \mathbb{R}$ is defined by $\|f\|_{1}=\int_{a}^{b}|f(t)| d t$. For a matrix $Q \in \mathbb{R}^{m \times n}$, the induced matrix norm is defined by $\|Q\|_{m}=\sqrt{\lambda_{\max }\left(Q^{T} Q\right)}$, where $\lambda_{\max }$ is the maximum eigenvalue of $Q^{T} Q$.

\section{PROBLEM STATEMENT}

In this work the following switched LPV system is considered $^{1}$ :

$$
\begin{aligned}
y^{(n)}(t)=-\sum_{i=0}^{n-1} & a_{i}(\sigma(t), \rho(t)) y^{(i)}(t) \\
& +\sum_{j=0}^{m} b_{j}(\sigma(t), \rho(t)) u^{(j)}(t)+\epsilon(t),
\end{aligned}
$$

where $y, u \in \mathbb{R}$, are the output and the input, respectively, generated by the switched LPV system. The term $\epsilon(t) \in \mathcal{L}_{\infty}$ represents uncertainties, disturbances and/or high frequency perturbations (possible effects of the noise in the input/output reflected in $\left.y^{(n)}(t)\right)$ in the system, and it is considered that the following assumption holds:

Assumption 1 The uncertain term $\epsilon(t)$ is essentially bounded, i.e. $\|\epsilon\| \leq \epsilon^{+}$, where $\epsilon^{+}$is a known positive constant.

The so-called "discrete state" $\sigma(t): \mathbb{R}^{+} \rightarrow \mathcal{Q}=$ $\{1, \ldots, q\}$ determines the current system dynamics among the possible $q$ "operating modes". The

\footnotetext{
1 In the literature there exist works dealing with the transformation of time varying systems to canonical forms, as in (1), see, e.g. [20] and [26].
} 
unknown scheduling parameter is represented by $\rho(t): \mathbb{R}^{+} \rightarrow \Theta \subseteq \mathbb{R}$ and it belongs to $\mathcal{L}_{\infty}$. The parameters $a_{0}: \mathcal{Q} \times \Theta \rightarrow I_{a_{0}}^{\sigma} \subseteq \mathbb{R}, a_{1}: \mathcal{Q} \times \Theta \rightarrow I_{a_{1}}^{\sigma} \subseteq \mathbb{R}, \ldots$, $a_{n-1}: \mathcal{Q} \times \Theta \rightarrow I_{a_{n-1}}^{\sigma} \subseteq \mathbb{R}$, and $b_{0}: \mathcal{Q} \times \Theta \rightarrow I_{b_{0}}^{\sigma} \subseteq \mathbb{R}$, $b_{1}: \mathcal{Q} \times \Theta \rightarrow I_{b_{1}}^{\sigma} \subseteq \mathbb{R}, \ldots, b_{m}: \mathcal{Q} \times \Theta \rightarrow I_{b_{m}}^{\sigma} \subseteq \mathbb{R}$ are unknown, where $I_{(\cdot)}^{\sigma}$ are given compact sets. The parameters $\left\{a_{i}(\sigma, \rho(t))\right\}_{i=0}^{n-1}$ and $\left\{b_{j}(\sigma, \rho(t))\right\}_{j=0}^{m}$ evolve in a compact set $\Omega^{\sigma}=I_{a_{0}}^{\sigma} \times \ldots \times I_{a_{n-1}}^{\sigma} \times I_{b_{0}}^{\sigma} \times \ldots \times I_{b_{m}}^{\sigma}$, which changes when $\sigma$ takes different values in $\mathcal{Q}$. In the following, it is assumed that time-varying vector of scheduling parameters is not accessible for measurements and only the domains $\Omega^{\sigma}$ are known for every value of $\sigma$ in $\mathcal{Q}$. The aim of this paper is to estimate the discrete state $\sigma(t)$ by the measurements of the output $y(t)$ and input $u(t)$. Then, estimate the continuous state, i.e. $y^{(k)}$ for $k=0,1, \ldots, n-1$.

\subsection{Preliminaries}

Define the parameter vector for the discrete state $\sigma$ as $\theta(\sigma, \rho(t))=\left[a_{0}(\cdot), \ldots, a_{n-1}(\cdot), b_{0}(\cdot), \ldots, b_{m}(\cdot)\right]^{T} \in \Omega^{\sigma}$. Some definitions for distinguishability and observability of the discrete state are introduced in the following.

Definition 1 Distinguishability of the system. The system (1) is called distinguishable, if for every pair $(i, j)$, the identity $y(t, \theta(i, \rho(t)))=y(t, \tilde{\theta}(j, \rho(t))), \forall t \in\left[t_{1}, t_{2}\right]$, implies that $i=j$, and $\theta, \tilde{\theta} \in \Omega^{i}$, for a given input $u$ and $i, j \in \mathcal{Q}$, for $\forall t \in\left[t_{1}, t_{2}\right]$.

Definition 2 Observability of the discrete state. The discrete state $\sigma(t)$ is observable if the system (1) is distinguishable, and $\theta(\sigma(t), \rho(t)) \notin\left(\Omega^{1} \cap \cdots \cap \Omega^{q}\right)$ during any time interval (see Fig. 1).

Fig. 1 depicts the discrete state observability definition. Note that $\theta(\sigma(t), \rho(t))$ may cross the set $\left(\Omega^{1} \cap \cdots \cap \Omega^{q}\right)$ for an instant of time, e.g. due to the switchings, but while $\theta(\sigma(t), \rho(t))$ does not remain in $\left(\Omega^{1} \cap \cdots \cap \Omega^{q}\right)$ during any time interval, the discrete state will be observable (see Fig. 1 left top case). On the contrary, if $\theta(\sigma(t), \rho(t))$ belongs to $\left(\Omega^{1} \cap \cdots \cap \Omega^{q}\right)$ during a time interval (as in Fig. 1 right top case), the discrete state will not be observable over this time interval.

\subsection{Description of the Proposed Solution}

The system (1) can be written in the regressor form as

$$
y^{(n)}(t)=\theta(\sigma(t), \rho(t)) \phi(t)+\epsilon(t),
$$

where $\theta(\cdot) \in \mathbb{R}^{1 \times r}$ is the unknown varying parameter vector, and $\phi(t)=\left[y(t), \cdots, y^{(n-1)}(t), u(t), \cdots\right.$, $\left.u^{(m)}(t)\right]^{T} \in \mathbb{R}^{r \times 1}$ is the regressor vector, where $r=$ $n+m+1$. It is considered that the following assumption holds.
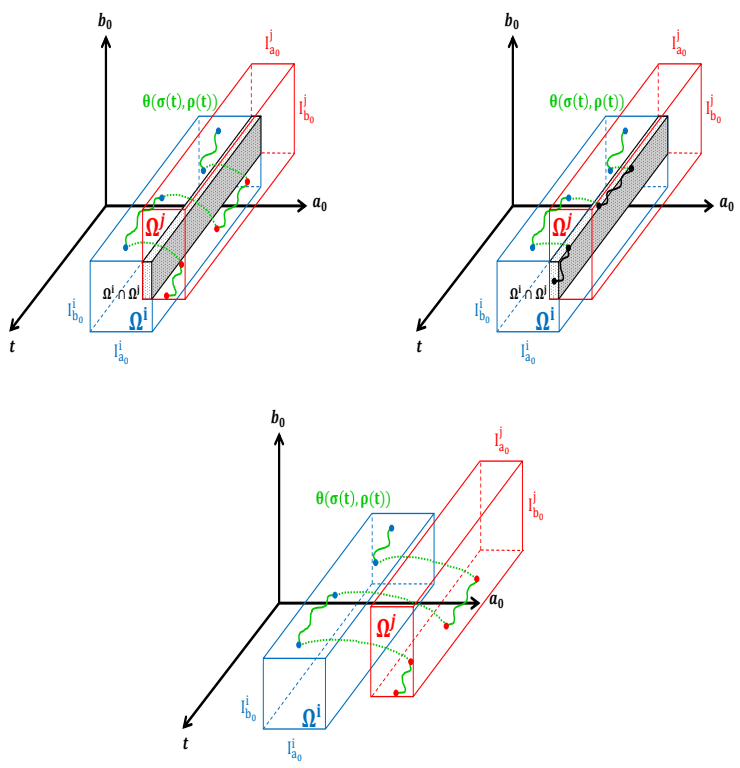

Fig. 1. Observability of the discrete state. From the geometric point of view, it is easy to see that the discrete state is observable for every $\theta$, if $\Omega^{i} \cap \Omega^{j}=\emptyset$ (the trivial case - bottom graph). The first two graphs correspond to the case $\Omega^{i} \cap \Omega^{j} \neq \emptyset$. The top one illustrates an observable discrete state case with non-empty intersections, i.e. $\theta(\sigma(t), \rho(t)) \notin\left(\Omega^{i} \cap \Omega^{j}\right)$ during any time interval, and the middle one depicts an unobservable discrete state case.

Assumption 2 The time derivative of the parameter vector is essentially bounded, i.e. $\|\dot{\theta}(\sigma(t), \rho(t))\|_{2} \leq \epsilon_{\theta}$, for almost all $t \geq 0$, with a known positive constant $\epsilon_{\theta}$.

The discrete state estimation problem can be solved if it is possible to identify the unknown parameter vector $\theta(\sigma(t), \rho(t))$ under the assumption that the domains $\Omega^{\sigma}$ for all $\sigma \in \mathcal{Q}$ are known and the conditions of Definitions 1 and 2 are satisfied for all $t \geq 0$.

Remark 1 Note that the main objective is to realize a good discrete state estimation and to do that it is not needed to identify the parameters exactly. It is only necessary to provide an approximate estimation with a sufficiently small error that allows to distinguish between the discrete states (see an example in Fig. 2). However, a large error in the parameter identification could yield a considerable error in the continuous state estimation.

\section{DISCRETE STATE ESTIMATION}

In the literature there exist many methods to identify constant and varying parameters, and the most popular ones belong to the group of least squares (LS) methods (e.g., non-recursive method of LS, recursive methods of LS, method of weighted LS, exponential forgetting with constant forgetting factor, exponential forgetting with variable forgetting factor, etc.). There exist 


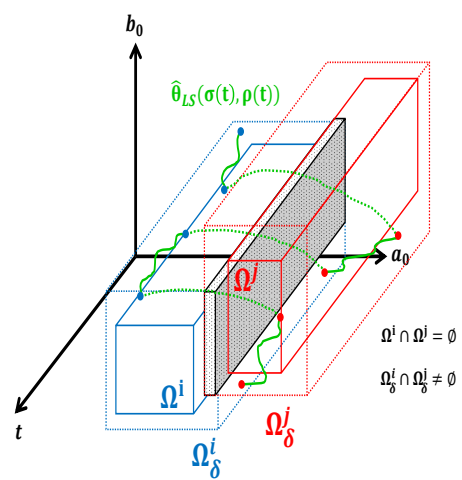

Fig. 2. Distinguishable sets $\Omega^{\sigma(t)}$ with error. In the graph the maximum parameter identification error is represented by the variable $\delta$ and the parameter estimation by $\hat{\theta}_{L S}$, so the estimation process will allow us to identify the set of parameters with a bounded estimation error of order $\delta$ (sets $\Omega_{\delta}^{i}$ and $\left.\Omega_{\delta}^{j}\right)$, that is represented by the volume enclosed by dotted line. Note that $\Omega^{i} \cap \Omega^{j}=\emptyset$ while $\Omega_{\delta}^{i} \cap \Omega_{\delta}^{j} \neq \emptyset$. However, since $\hat{\theta}_{L S}(\sigma(t), \rho(t)) \notin \Omega_{\delta}^{i} \cap \Omega_{\delta}^{j}$ it is possible to estimate the discrete state.

also many modifications of the LS methods (e.g., method of generalized LS, method of extended LS, method of bias correction, instrumental variables method, etc.), and bayesian method, maximum likelihood methods, extended Kalman filter, modulating functions methods, subspace methods, etc. (see, e.g., [8] and [14]).

Note that to solve the formulated problem in this paper, it is possible to use any method that allows us to identify the parameters with such a sufficiently small error that it is possible to distinguish the discrete state $\sigma(t)$. It is necessary to mention that the objective is not to realize a good parameter identification for $\theta(\cdot)$ but a good estimation of $\sigma(t)$. In the following, the used parameter identification method is described.

\subsection{Description of the Parameter Identification}

Consider system (2) and apply the mean value theorem to each entry of $\theta(\sigma(t), \rho(t))$. Then, (2) can be rewritten, for all $t \in\left[t_{0}, t_{0}+T\right]$, as:

$$
y^{(n)}(t)=\left[\theta\left(t_{0}\right)+\left(t-t_{0}\right) \dot{\theta}(c(t))\right] \phi(t)+\epsilon(t),
$$

where $\dot{\theta}(c(t))=\left[\dot{a}_{0}\left(c_{0}(t)\right), \dot{a}_{1}\left(c_{1}(t)\right), \ldots, \dot{b}_{m}\left(c_{r}(t)\right)\right]$, $\left\{c_{i}(t)\right\}_{i=1}^{r} \in\left(t_{0}, t_{0}+T\right)$ represent time varying values for each component of $\theta(\sigma(t), \rho(t)), \theta\left(t_{0}\right)=$ $\theta\left(\sigma\left(t_{0}\right), \rho\left(t_{0}\right)\right)$, and $T$ is a positive constant that defines a time window in which it is expected that the parameter identification is carried out. Note that if the derivatives of the output $y(t)$ and the input $u(t)$ are directly measurable, these values can be entered directly into the regressor vector $\phi(t)$ and therefore the classical LS method can be applied directly to estimate $\theta\left(t_{0}\right)$ on $\left[t_{0}, t_{0}+T\right]$. However, the derivatives are not measurable, the parameters are time variant, and there exist some uncertainties, disturbances or/and noise. Therefore, some considerations have to be made.

Firstly, in order to avoid the direct calculation of the derivatives of the output and input, the state variable filter approach is used [8]. The main idea is to filter the output signal $y(t)$ and input signal $u(t)$ with the same stable Butterworth low-pass filter ${ }^{2}$ that has the following structure in the Laplace domain:

$$
F(s)=\frac{1}{1+\lambda_{1} s+\cdots+\lambda_{p} s^{p}},
$$

where $p=\max (n, m+1)$, and the design of the filter parameters $\lambda_{k}$ is free (stable filter). For example, one can set the design parameters so that $F(s)$ has an arbitrary cut-off frequency $\omega_{c}$, and the form:

$$
F(s)=\frac{1}{\prod_{j}\left(1+\alpha_{j} \frac{s}{\omega_{c}}+\beta_{j} \frac{s^{2}}{\omega_{c}^{2}}\right)},
$$

with the coefficients being:

- Even $p$ : $\left\{\alpha_{j}\right\}_{j=1}^{p / 2}=\left\{2 \cos \frac{(2 j-1) \pi}{2 p}\right\}_{j=1}^{p / 2},\left\{\beta_{j}\right\}_{j=1}^{p / 2}=1$.

- Odd $p: \alpha_{1}=1,\left\{\alpha_{j}\right\}_{j=2}^{(p+1) / 2}=\left\{2 \cos \frac{(j-1) \pi}{p}\right\}_{j=2}^{(p+1) / 2}$, $\beta_{1}=0,\left\{\beta_{j}\right\}_{j=2}^{(p+1) / 2}=1$.

After filtering, system (3), for all $t \in\left[t_{0}, t_{0}+T\right]$, becomes:

$$
Y(t)=\theta\left(t_{0}\right) \Psi(t)+\Theta(t)+\Xi(t)
$$

where $Y(t)=f(t) * y^{(n)}(t), \Psi(t)=f(t) * \phi(t), \Theta(t)=$ $f(t) *\left(\left(t-t_{0}\right) \dot{\theta}\left(\sigma\left(c_{i}(t)\right), \rho\left(c_{i}(t)\right)\right) \phi(t)\right)$, and $\Xi(t)=$ $f(t) * \epsilon(t)$, where $f(t)$ is the impulse response of the filter (4). If it is assumed that the uncertain terms $\Theta(t)$ and $\Xi(t)$, are equal to zero, then, post-multiplying by $\Psi^{T}(t)$ and integrating, it is obtained

$$
\int_{t_{0}}^{t_{0}+T} Y(\tau) \Psi^{T}(\tau) d \tau=\theta\left(t_{0}\right) \int_{t_{0}}^{t_{0}+T} \Psi(\tau) \Psi^{T}(\tau) d \tau
$$

where $T$ is a positive constant that defines the time window of identification. Thus, the classical LS Method can be applied to estimate the term $\theta\left(t_{0}\right)$, i.e.

$$
\hat{\theta}_{L S}\left(t_{0}\right)=\int_{t_{0}}^{t_{0}+T} Y(\tau) \Psi^{T}(\tau) d \tau \Upsilon^{-1}
$$

\footnotetext{
2 One can choose a Butterworth low-pass filter or any other type of low-pass filter, e.g. Bessel, Chebyshev, etc. (see, e.g. $[5])$.
} 
with $\Upsilon=\int_{t_{0}}^{t_{0}+T} \Psi(\tau) \Psi^{T}(\tau) d \tau$. In order to implement (6) it is necessary to construct the new regressor vector $\Psi(t)$ and the term $Y(t)$, with this aim, the state variable filter approach is used.

\subsubsection{State Variable Filter Design.}

The principle of state variable filters is to use a low-pass filter that damps out the higher frequency noise and transform it into a state space representation, such that the states are the derivatives of the filter output, i.e. the filtered signal. They are designed to suppress any noise above the cut-off frequency.

Consider that the regressor vector has the following structure

$$
\Psi(t)=\left[y_{f_{0}}(t), \ldots, y_{f_{n-1}}(t), u_{f_{0}}(t), \ldots, u_{f_{m}}(t)\right]^{T}
$$

The elements of the vector $\Psi(t)$ can be easily obtained as the state variables of the following state space systems, which represent the controllable canonical form of the filter (4), i.e.

$$
\begin{aligned}
& \dot{y}_{F}(t)=A y_{F}(t)+b y(t), \\
& \dot{u}_{F}(t)=A u_{F}(t)+b u(t),
\end{aligned}
$$

where $y_{F}(t)=\left[y_{f_{0}}(t), \ldots, y_{f_{p-1}}(t)\right]^{T} \in \mathbb{R}^{p}$ and $u_{F}(t)=\left[u_{f_{0}}(t), \ldots, u_{f_{p-1}}(t)\right]^{T} \in \mathbb{R}^{p}$ and

$$
A=\left[\begin{array}{ccccc}
0 & 1 & 0 & \cdots & 0 \\
0 & 0 & 1 & \cdots & 0 \\
\vdots & \vdots & \vdots & \ddots & \vdots \\
0 & 0 & 0 & \cdots & 1 \\
-\frac{1}{\lambda_{p}} & -\frac{\lambda_{1}}{\lambda_{p}} & -\frac{\lambda_{2}}{\lambda_{p}} & \cdots & -\frac{\lambda_{p-1}}{\lambda_{p}}
\end{array}\right], b=\left[\begin{array}{c}
0 \\
0 \\
\vdots \\
0 \\
\frac{1}{\lambda_{p}}
\end{array}\right] .
$$

Note that, since $\dot{y}_{f_{p-1}}(t)=y_{f_{p}}(t), \dot{y}_{f_{p}}(t)$ can be constructed as follows:

$$
\dot{y}_{f_{p}}(t)=\left[-\frac{1}{\lambda_{p}},-\frac{\lambda_{1}}{\lambda_{p}}, \ldots,-\frac{\lambda_{p-1}}{\lambda_{p}}\right] y_{F}(t)+\frac{1}{\lambda_{p}} y(t) .
$$

For the linear time-invariant systems (7), in the same way for (8); assuming that the filter initial conditions are equal to zero, it is possible to express the $n$ and $m+1$ transfer functions, respectively, as follows:

$$
\begin{aligned}
Y_{f_{i}}(s) & =\frac{s^{i}}{1+\lambda_{1} s+\cdots+\lambda_{p} s^{p}} Y(s), i=0, \ldots, n-1 . \\
U_{f_{j}}(s) & =\frac{s^{j}}{1+\lambda_{1} s+\cdots+\lambda_{p} s^{p}} U(s), j=0, \ldots, m .
\end{aligned}
$$

In order to calculate the filter transient time $\xi$ is necessary to consider the Laplace transformation existence of the corresponding input filter, i.e. the convergence of $\int_{0}^{\infty}|y(t)| e^{-\alpha t} d t$, for $\alpha>0$. It is well-known that this integral converges if $|y(t)|<\Gamma e^{\varrho t}, \forall t>0$, some $\Gamma>0$, and $\alpha>\varrho$; in the same way for $u(t)$. In this sense, if the corresponding filter input satisfies such a condition then the following procedure is proposed to calculate the filter transient time:

1. Design the parameters for filter (4).

2. Express the filter inputs, i.e. $y(t)$ and $u(t)$, in the Laplace domain.

3. Obtain the corresponding transfer functions, i.e. $Y_{f_{0}}(s), \ldots, Y_{f_{n-1}}(s)$ and $U_{f_{0}}(s), \ldots, U_{f_{m}}(s)^{3}$.

4. Obtain the partial fraction expansion for each transfer function.

5. Apply the inverse Laplace transformation for each transfer function.

6. Identify the transient part for each solution $y_{f_{0}}(t), \ldots$, $y_{f_{n-1}}(t)$ and $u_{f_{0}}(t), \ldots, u_{f_{m}}(t)$, respectively.

7. Calculate the transient time ${ }^{4}$ for each solution, i.e. $\xi_{y_{0}}, \ldots, \xi_{y_{n-1}}$ and $\xi_{u_{0}}, \ldots, \xi_{u_{m}}$, assuming a tolerance band.

8. Define $\xi$ as the slowest transient time.

Otherwise:

1. Design the parameters for filter (4).

2. Simulate the state space systems (7) and (8) with their corresponding inputs.

3. Calculate by simulation the transient time for each solution, i.e. $\xi_{y_{0}}, \ldots, \xi_{y_{n-1}}$ and $\xi_{u_{0}}, \ldots, \xi_{u_{m}}$, assuming a tolerance band.

4. Define $\xi$ as the slowest transient time.

Note that it is possible to modify the corresponding transient times by adjusting the filter parameters (analytically or by simulation). However, there will be a tradeoff between the desired transient time and the corresponding cut-off frequency. It is also worth highlighting two points of the first procedure: 1) this procedure can be applied for any filter input signal with a Laplace domain representation, and 2) most of these signals, for conventional parametric identification, are sinusoidal. Moreover, most of the physically feasible inputs can be expressed as a sum of the basic signals which have a representation in the Laplace domain, i.e. step, ramp,

\footnotetext{
3 The state space systems (7)-(8) provide $p$ elements, i.e. $y_{f_{0}}(t), \ldots, y_{f_{p-1}}(t)$, and $u_{f_{0}}(t), \ldots, u_{f_{p-1}}(t)$, respectively. Nevertheless, only the first $n$ and $m+1$ elements will be taken to construct the regressor vector $\Psi(t)$.

4 This transient time does not correspond with the definition of settling time given for linear systems with constant inputs but, for this case, it is possible to calculate it analytically since in general $y_{F}(t)=e^{A t} y_{F_{t}}(t)+y_{F_{p}}(t)$, where $e^{A t} y_{F_{t}}(t)$ represents the transient of the solution, and $y_{F_{p}}(t)$ the permanent part, i.e. the filtered signal; in the same way for $u_{F}(t)$.
} 
parabola and sinusoidal signals. If this is not the case, it is possible to adjust $\xi$ by trial and error method.

Let us suppose that the following assumptions are satisfied.

Assumption 3 The regressor vector $\phi$ is essentially bounded, i.e. $\|\phi(t)\|_{2} \leq \phi^{+}$, for almost all $t \geq 0$, with a known constant $\phi^{+}>0$.

Assumption 4 The vector $\Psi(t)$ satisfies the following persistent excitation condition:

$$
\eta_{2} I \geq \frac{1}{T} \int_{t_{0}}^{t_{0}+T} \Psi(\tau) \Psi^{T}(\tau) d \tau \geq \eta_{1} I
$$

for some scalars $0<\eta_{1} \leq \eta_{2}$, for some $T>0$, and all $t_{0} \geq 0$.

Assumptions 3 and 4 make reference to the excitation level. The level of excitation can be calculated on-line by means of many excitation signals which have been extensively studied in the literature (see, e.g. [14]). Now the following theorem about parameter identification is established.

Theorem 1 Consider system (5) and the LS method (6). Let Assumptions 1-4 be satisfied. Then, after the transient time $\xi$, the parameter estimation error is bounded, i.e.

$$
\left\|\tilde{\theta}\left(t_{0}\right)\right\|_{2} \leq \frac{T\|f\|_{1}\|\Psi\|_{2}}{\eta_{2}}\left(\epsilon_{\theta} \phi^{+}+\frac{\epsilon^{+}}{T}\right)
$$

where $\tilde{\theta}(\cdot)=\hat{\theta}_{L S}\left(t_{0}\right)-\theta\left(t_{0}\right)$

Proof. The proof is constructive. Post-multiplying (5) by $\Psi(t)$ and integrating, it is obtained

$$
\begin{gathered}
\int_{t_{0}}^{t_{0}+T} Y(\tau) \Psi^{T}(\tau) d \tau=\theta\left(t_{0}\right) \int_{t_{0}}^{t_{0}+T} \Psi(\tau) \Psi^{T}(\tau) d \tau \\
\quad+\int_{t_{0}}^{t_{0}+T} \Theta(\tau) \Psi^{T}(\tau) d \tau+\int_{t_{0}}^{t_{0}+T} \Xi(\tau) \Psi^{T}(\tau) d \tau .
\end{gathered}
$$

Solving for $\theta\left(t_{0}\right)$, it gives

$$
\begin{array}{r}
\theta\left(t_{0}\right)=\left[\int_{t_{0}}^{t_{0}+T} Y(\tau) \Psi^{T}(\tau) d \tau-\int_{t_{0}}^{t_{0}+T} \Theta(\tau) \Psi^{T}(\tau) d \tau\right. \\
\left.-\int_{t_{0}}^{t_{0}+T} \Xi(\tau) \Psi^{T}(\tau) d \tau\right] \Upsilon^{-1}
\end{array}
$$

By comparing (6) and (12) the estimation error $\tilde{\theta}\left(t_{0}\right)$ has the following form:

$$
\begin{aligned}
\tilde{\theta}\left(t_{0}\right)=\int_{t_{0}}^{t_{0}+T} \Theta(\tau) \Psi^{T}(\tau) d \tau \Upsilon^{-1} & \\
& \quad+\int_{t_{0}}^{t_{0}+T} \Xi(\tau) \Psi^{T}(\tau) d \tau \Upsilon^{-1},
\end{aligned}
$$

The norm of the estimation error is given by:

$$
\begin{aligned}
\left\|\tilde{\theta}\left(t_{0}\right)\right\|_{2} \leq & \int_{t_{0}}^{t_{0}+T}\|\Theta(\tau)\|_{2}\left\|\Psi^{T}(\tau)\right\|_{2} d \tau\|\Upsilon\|_{m}^{-1} \\
& +\int_{t_{0}}^{t_{0}+T}\|\Xi(\tau)\|_{2}\left\|\Psi^{T}(\tau)\right\|_{2} d \tau\|\Upsilon\|_{m}^{-1}
\end{aligned}
$$

Taking into account that the term $\Psi$ is known or measurable, its norm can be easily calculated. Moreover, considering the fact that $\|f * g\|_{2} \leq\|f\|_{1}\|g\|_{2}$ (see, e.g. [2]), if the Assumption 4 holds, it will be obtained that

$$
\left\|\tilde{\theta}\left(t_{0}\right)\right\|_{2} \leq \frac{T}{\eta_{2}}\|f\|_{1}\|\dot{\theta}\|_{2}\|\phi\|_{2}\|\Psi\|_{2}+\frac{1}{\eta_{2}}\|f\|_{1}\|\epsilon\|_{2}\|\Psi\|_{2}
$$

Considering the bounds for $\dot{\theta}(\cdot)$ and $\epsilon(t)$, and Assumption $3,\left\|\tilde{\theta}\left(t_{0}\right)\right\|$ can be written as

$$
\left\|\tilde{\theta}\left(t_{0}\right)\right\|_{2} \leq \frac{T\|f\|_{1}\|\Psi\|_{2}}{\eta_{2}}\left(\epsilon_{\theta} \phi^{+}+\frac{\epsilon^{+}}{T}\right) .
$$

Thus, theorem 1 is proven.

\subsection{Parameter Identification on the Switching Times}

It is clear that during the time interval $\left[\xi, t_{1}\right)$ the established in the Theorem 1 is true. Nevertheless, on the switching times the condition $\|\dot{\theta}\|_{2} \leq \epsilon_{\theta}$, from Assumption 2, may not be valid, so the estimation error can be higher. However, it is possible to consider the switches (the jumps in the parameter vector) as the beginning of a new process or a change in the initial conditions. Therefore, when a switch happens it is necessary to wait again $T+\xi$ units of time so that the Theorem 1 and its conditions are satisfied and to ensure again that the parameter identification error is bounded by (11).

Taking into account previous explanations the following minimal dwell time definition and assumption are established.

Definition 3 The minimal dwell time is a constant $T_{\delta}>$ 0 . A switching signal is said to satisfy the minimal dwell time property if switching times $t_{1}, t_{2}, \ldots$ fulfill the inequality $t_{j+1}-t_{j} \geq T_{\delta}$ for all $j \geq 1$ [11]. 
Assumption 5 Assume that the signal $\sigma(t)$ satisfies the minimal dwell time property, such that $t_{j+1}-t_{j} \geq T_{\delta}>$ $T+\xi+\zeta$ for all $j \geq 1$, with a small positive constant $\zeta$.

\subsection{Discrete State Estimation}

Once the parameters estimation is obtained with its corresponding error bound it is possible to use this information to estimate the discrete state. The Algorithm 1 describes the way in which the discrete state is estimated.

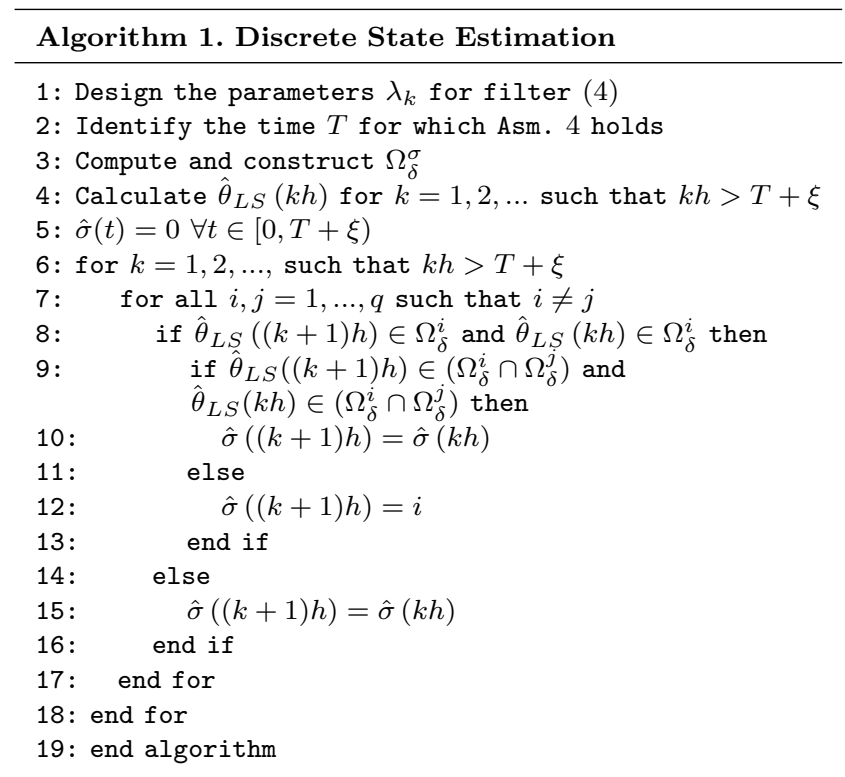

Remark 2 In Algorithm 1, the sample time is represented by $h$. The domains $\Omega_{\delta}^{\sigma}$, for each $\sigma=1, \ldots, q$, are defined as follows (see Fig. 3), while $\Omega_{\delta}^{\sigma}=\left(I_{a_{0}}^{\sigma}+[-\delta, \delta]\right) \times$ $\ldots \times\left(I_{a_{n-1}}^{\sigma}+[-\delta, \delta]\right) \times\left(I_{b_{0}}^{\sigma}+[-\delta, \delta]\right) \times \ldots \times\left(I_{b_{m}}^{\sigma}+[-\delta, \delta]\right)$, with $\delta=\|\tilde{\theta}\|_{\infty} \leq\|\tilde{\theta}\|_{2} / \sqrt{r}$ (the maximum parameter identification error), where $r=n+m+1$.

Note that if the Assumption 5 is satisfied and the discrete state $\sigma(t)$ is observable according to Definition 2, then the Algorithm 1 provides a finite time estimation. Moreover, if $\hat{\theta}_{L S}(\sigma(t), \rho(t)) \notin \Omega_{\delta}^{i} \cap \Omega_{\delta}^{i}$, an exact discrete state estimation is achieved.

\subsubsection{Discussion about the algorithm}

1. The discrete state estimation is carried out by means of the parameter estimation $\hat{\theta}_{L S}(\cdot)$ and the knowledge of the sets $\Omega^{\sigma}$. However, the key point for discrete state estimation consists of the sets $\Omega_{\delta}^{\sigma}$ generated due to the identification error. It has already been defined that the discrete state is observable if every pair $(i, j)$ of systems is distinguishable and $\theta(\sigma(t), \rho(t)) \notin$ $\left(\Omega^{1} \cap \cdots \cap \Omega^{q}\right)$ during any time interval.

Nevertheless, since $\hat{\theta}_{L S}(\cdot)$ is used to estimate the discrete state, now it is necessary to verify that

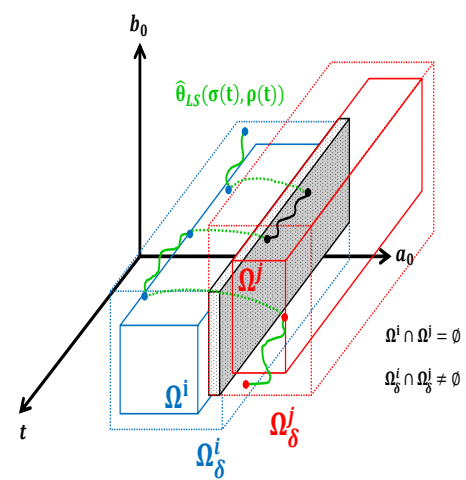

Fig. 3. Discrete state estimation. Note that, even though $\Omega^{i} \cap \Omega^{j}=\emptyset$, the error might produce an intersection $\Omega_{\delta}^{i} \cap \Omega_{\delta}^{j} \neq \emptyset$. However, with only one parameter being out of such an intersection, it will be sufficient to estimate the discrete state correctly.

$\hat{\theta}_{L S}(\cdot) \notin\left(\Omega_{\delta}^{1} \cap \cdots \cap \Omega_{\delta}^{q}\right)$ during any time interval (see Fig. 2). Note that it is not possible to predict if such a condition will hold, but it is possible to check it online. In this way, Algorithm 1 foresees such a situation and when $\hat{\theta}_{L S}(\cdot) \in\left(\Omega_{\delta}^{1} \cap \cdots \cap \Omega_{\delta}^{q}\right)$ holds in some time intervals (see Fig. 3), the last correct value $\hat{\sigma}(k h)$ is assigned to the next value $\hat{\sigma}((k+1) h)$. It is clear that when $\hat{\theta}_{L S}(\cdot) \in\left(\Omega_{\delta}^{1} \cap \cdots \cap \Omega_{\delta}^{q}\right)$ in some time intervals ${ }^{5}$, Algorithm 1 does not guarantee a correct discrete state estimation but neither assures a wrong estimation.

2. An example of the estimation that provides Algorithm 1 is the one that appears in Fig. 4. Note that it is only possible to ensure that the discrete state estimation is correct after $T+\xi+\zeta$ units of time, i.e. the sum of the time identification window plus the filter transient time plus a small positive constant ${ }^{6}$. This is due to the fact that if a switching takes place inside an interval $\left[t_{j}, t_{j}+T+\xi\right)$, the parameter identification is in general not correct. Therefore, the assumption on the minimum dwell time had to be considered.

3. Due to one of the objectives is to obtain a practical solution, a simple algorithm is provided. Therefore, the main advantage of the given method is its simplicity. Note that simplicity is not a shortage, it is exactly the inverse since it is possible to obtain a good result without need to use a more complex method.

5 It is worth saying it is enough that only one element of $\hat{\theta}_{L S}(\cdot)$ is out of the intersection so that the condition $\hat{\theta}_{L S}(\cdot) \notin\left(\Omega_{\delta}^{1} \cap \cdots \cap \Omega_{\delta}^{q}\right)$ is fulfilled. Therefore, if the parameter estimation error is sufficiently small and just one parameter allows us to satisfy such a condition, the estimation of the discrete state will be achieved. From this point of view, such a condition is not so restrictive.

${ }^{6}$ For the purpose of implementation, based on Algorithm 1 and dwell time property, it is considered that $\zeta$ is three times the sampling time, i.e. $\zeta=3 h$. 


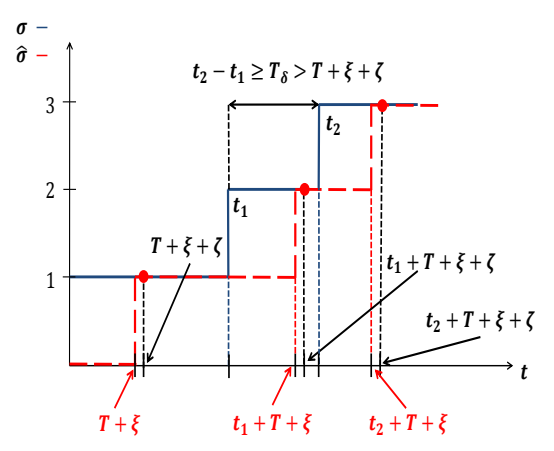

Fig. 4. Dwell time. Note that when a change between sets $\Omega_{\delta}^{\sigma}$ is detected the discrete state estimation is maintained in the past value.

\section{CONTINUOUS STATE ESTIMATION}

Suppose that system (1) is given in the form

$$
\begin{aligned}
y^{(n)}(t)=-\sum_{i=0}^{n-1} a_{i}(\sigma(t), \rho(t)) y^{(i)}(t) & \\
& +b_{0}(\sigma(t), \rho(t)) u(t)+\epsilon(t),
\end{aligned}
$$

where $\epsilon(t)=\sum_{i=0}^{n-1} a_{i}^{\delta} y^{(i)}(t)+b_{0}(\sigma(t), \rho(t)) \nu_{1}(t)$, with $a_{0}^{\delta}, a_{1}^{\delta}, \ldots, a_{n-1}^{\delta}$ representing some constant parameter uncertainties with respect to the nominal values $a_{0}(\cdot), a_{1}(\cdot), \ldots, a_{n-1}(\cdot)$, and $\nu_{1}(t) \in \mathcal{L}_{\infty}$ an input noise satisfying $\left\|\nu_{1}\right\| \leq \nu^{+}$, with $\nu^{+}$a known positive constant. Based on the realization theory of observability canonical forms, an equivalent state-space form can be written as follows

$$
\begin{aligned}
& \dot{x}=A(t) x+A^{\delta} x+B(t)\left(u+\nu_{1}\right), \\
& y=C x, \bar{y}=y+\nu_{2},
\end{aligned}
$$

where $x \in \mathbb{R}^{n}, y, u \in \mathbb{R}$ are the state vector, the output and the input, respectively. For sake of readability, from now onward $A(t)=A(\sigma(t), \theta(t))$ and $B(t)=$ $B(\sigma(t), \theta(t))$. It is considered that $\bar{y} \in \mathbb{R}$ is the available measurable output with the noise $\nu_{2}(t)$ satisfying $\left\|\nu_{2}\right\| \leq$ $\nu^{+}$, just like the input noise. The matrix $A^{\delta}$ contains the constant parameter uncertainties $a_{0}^{\delta}, a_{1}^{\delta}, \ldots, a_{n-1}^{\delta}$. Matrices $A(t), A^{\delta}, B(t)$ and $C$ have corresponding dimensions and the following structure:

$$
A(t)=\left[\begin{array}{ccccc}
0 & 1 & 0 & \cdots & 0 \\
0 & 0 & 1 & \cdots & 0 \\
\vdots & \vdots & \vdots & \ddots & \vdots \\
0 & 0 & 0 & \cdots & 1 \\
-a_{0}(\cdot) & -a_{1}(\cdot) & -a_{2}(\cdot) & \cdots & -a_{n-1}(\cdot)
\end{array}\right],
$$

$$
\begin{gathered}
A^{\delta}=\left[\begin{array}{ccccc}
0 & 0 & 0 & \cdots & 0 \\
0 & 0 & 0 & \cdots & 0 \\
\vdots & \vdots & \vdots & \ddots & \vdots \\
0 & 0 & 0 & \cdots & 0 \\
a_{0}^{\delta} & a_{1}^{\delta} & a_{2}^{\delta} & \cdots & a_{n-1}^{\delta}
\end{array}\right], B(t)=\left[\begin{array}{c}
0 \\
0 \\
\vdots \\
b_{0}(\cdot)
\end{array}\right], \\
C=\left[\begin{array}{llll}
1 & 0 & \cdots & 0
\end{array}\right] .
\end{gathered}
$$

It is clear that the state-space realization (14)-(15) is in the observable canonical form. Now, let us describe the continuous state observer design. Consider the following hybrid observer

$$
\begin{aligned}
& \dot{\hat{x}}=\hat{A}(t) \hat{x}+\hat{B}(t) u+L(\hat{\sigma}(t))(\bar{y}-C \hat{x}), \\
& \hat{y}=C \hat{x}
\end{aligned}
$$

where $\hat{x} \in \mathbb{R}^{n}, \hat{y} \in \mathbb{R}$, the design matrix $L(\cdot) \in \mathbb{R}^{n \times 1}$, and the observer matrices, $\hat{A}(t)$ and $\hat{B}(t)$, are the estimated system matrices obtained by the parameter identification method. The following assumptions ensure the possibility for continuous state estimation.

Assumption 6 The matrices of the system (14) satisfy the following convex sum properties:

$$
\begin{gathered}
A(t)=\sum_{i=1}^{k} \mu_{i}^{a}(\sigma(t), \theta(t)) A_{i}^{\sigma(t)}, \\
\sum_{i=1}^{k} \mu_{i}^{a}(\sigma(t), \theta(t))=1, \\
B(t)=\sum_{i=1}^{k} \mu_{i}^{b}(\sigma(t), \theta(t)) B_{i}^{\sigma(t)}, \\
\sum_{i=1}^{k} \mu_{i}^{b}(\sigma(t), \theta(t))=1,
\end{gathered}
$$

where $\mu_{i}^{a}(\cdot), \mu_{i}^{b}(\cdot)$ are the unknown weighting functions, and $A_{i}^{\sigma(t)}, B_{i}^{\sigma(t)}$ are known constant matrices for each $\sigma$ and for all $\theta(t) \in\left(\Omega^{1} \cup \cdots \cup \Omega^{q}\right)$.

In order to deduce the matrices $A_{i}^{\sigma(t)}, B_{i}^{\sigma(t)}$ a convex polytopic method is used (see, e.g. the method given in [18], which allows us to embed LPV matrices into a convex polytope of constant matrices). This method computes the matrices $A_{i}^{\sigma(t)}, B_{i}^{\sigma(t)}$ only with the information on the given closed intervals $I_{(\cdot)}^{\sigma(t)}$.

Assumption 7 The parameter identification error on the system matrices is bounded, i.e.

$$
\left\|\Delta_{A}(t)\right\|_{m} \leq \delta_{A}, \quad\left\|\Delta_{B}(t)\right\|_{2} \leq \delta_{B}, \forall t \geq 0,
$$

where $\Delta_{A}(t)=A(t)-\hat{A}(t), \Delta_{B}(t)=B(t)-\hat{B}(t)$, and $\delta_{A}, \delta_{B}$ are known positive constants ${ }^{7}$.

\footnotetext{
7 Note that due to Theorem 1 Assumption 7 is satisfied. Moreover, the constants $\delta_{A}, \delta_{B}$ can be calculated based on the known parameter intervals $I_{a_{i}}^{\sigma}, I_{b_{i}}^{\sigma}$, and the parameter identification results.
} 
Taking into account the previous explanations, the following theorem can be stated:

Theorem 2 Let the observer (16)-(17) be applied to the system (14)-(15). Let Assumptions 6-7 be satisfied. If there exist positive definite symmetric matrices $Q_{\sigma}$ and $P_{\sigma}$ such that the following equations are satisfied for all $\sigma=1, \ldots, q$

$$
\begin{aligned}
& P_{\sigma}(\left.A_{i}^{\sigma}-L(\hat{\sigma}) C\right)+\left(A_{i}^{\sigma}-L(\hat{\sigma}) C\right)^{T} P_{\sigma} \\
& \quad+\frac{6}{\varpi_{\sigma}} P_{\sigma}^{2}+\varpi_{\sigma} \delta_{A}^{2} I=-Q_{\sigma}, \forall i=1, \ldots, k,
\end{aligned}
$$

where $\varpi_{\sigma}>0$ for each $\sigma$ and $\forall i=1, \ldots, k$. Then, the continuous state estimation error $e=x-\hat{x}$ converges exponentially to a neighborhood of the origin:

$$
\|e\|_{2} \leq \frac{\beta^{* 1 / 2}}{\left(\gamma_{v} P_{\min }^{*}\right)^{1 / 2}}
$$

where $\gamma_{v}=\frac{Q_{\min }^{*}}{P_{\max }^{*}}, Q_{\min }^{*}=\min _{\forall \sigma \in \mathcal{Q}}\left(\lambda_{\min }\left(Q_{\sigma}\right)\right), P_{\max }^{*}=$ $\max _{\forall \sigma \in \mathcal{Q}}\left(\lambda_{\max }\left(P_{\sigma}\right)\right), P_{\min }^{*}=\min _{\forall \sigma \in \mathcal{Q}}\left(\lambda_{\min }\left(P_{\sigma}\right)\right)$, and

$$
\begin{aligned}
\beta^{*}=\varpi^{*}\left[\left(\delta_{A}^{2}+\right.\right. & \left.\left\|A^{\delta}\right\|_{m}^{2}\right)\|x\|_{2}^{2}+\left(\delta_{B}\|u\|\right)^{2} \\
& \left.+\left(\|B(t)\|_{2}^{2}+\|L(\hat{\sigma})\|_{2}^{2}\right) \nu^{+^{2}}\right],
\end{aligned}
$$

where $\varpi^{*}=\max _{\forall \sigma \in \mathcal{Q}}\left(\varpi_{\sigma}\right)$.

Proof. The continuous state estimation error has the following structure

$$
\begin{aligned}
\dot{e}=A(t) x+A^{\delta} x- & \hat{A}(t) \hat{x}+B(t) \nu_{1}-L(\hat{\sigma}) \nu_{2} \\
& -L(\hat{\sigma}) C e+[B(t)-\hat{B}(t)] u,
\end{aligned}
$$

adding and subtracting $A(t) \hat{x}$, it is obtained

$$
\begin{aligned}
\dot{e}=[A(t) & -L(\hat{\sigma}) C] e+\Delta_{A}(t)(x-e) \\
& +A^{\delta} x+\Delta_{B}(t) u+B(t) \nu_{1}-L(\hat{\sigma}) \nu_{2},
\end{aligned}
$$

Take $V_{\sigma}=e^{T} P_{\sigma} e$. Note that $V_{\sigma}$ is positive definite and radially unbounded, i.e.

$$
P_{\min }^{*}\|e\|_{2}^{2} \leq V_{\sigma} \leq P_{\max }^{*}\|e\|_{2}^{2} .
$$

The time derivative of $V_{\sigma}$ along the trajectories of the system (21) is given by

$$
\begin{aligned}
& \dot{V}_{\sigma}=e^{T}\left\{P_{\sigma}[A(t)-L(\hat{\sigma}) C]\right. \\
& \left.+[A(t)-L(\hat{\sigma}) C]^{T} P_{\sigma}\right\} e+2 e^{T} P_{\sigma} \Delta_{A}(t)(x-e) \\
& \quad+2 e^{T} P_{\sigma} A^{\delta} x+2 e^{T} P_{\sigma} \Delta_{B}(t) u \\
& \quad+2 e^{T} P_{\sigma} B(t) \nu_{1}-2 e^{T} P_{\sigma} L(\hat{\sigma}) \nu_{2}
\end{aligned}
$$

From Assumption 7 and the well-known inequality $2 X^{T} Y \leq \frac{1}{\varpi} X^{T} X+\varpi Y^{T} Y$ for any scalar $\varpi>0$, recalling that $\left\|\nu_{1}\right\| \leq \nu^{+}$and $\left\|\nu_{2}\right\| \leq \nu^{+}$, it follows that

$$
\begin{gathered}
\dot{V}_{\sigma} \leq e^{T}\left\{P_{\sigma}[A(t)-L(\hat{\sigma}) C]+[A(t)-L(\hat{\sigma}) C]^{T} P_{\sigma}\right. \\
\left.+\frac{6}{\varpi_{\sigma}} P_{\sigma}^{2}+\varpi_{\sigma} \delta_{A}^{2} I\right\} e+\varpi_{\sigma}\left(\delta_{A}\|x\|_{2}\right)^{2} \\
+\varpi_{\sigma}\left(\left\|A^{\delta}\right\|_{m}\|x\|_{2}\right)^{2}+\varpi_{\sigma}\left(\delta_{B}\|u\|\right)^{2} \\
+\varpi_{\sigma}\left(\|B(t)\|_{2}^{2}+\|L(\hat{\sigma})\|_{2}^{2}\right) \nu^{+^{2}},
\end{gathered}
$$

From Assumption 6, (18) and (20), it follows that

$$
\begin{aligned}
& \dot{V}_{\sigma} \leq-\sum_{i=1}^{k} \sum_{j=1}^{k} \mu_{i}^{a}(\sigma(t), \theta(t)) \mu_{j}^{b}(\sigma(t), \theta(t)) e^{T} Q_{\sigma} e+\beta^{*} \\
& \dot{V}_{\sigma} \leq-e^{T} Q_{\sigma} e+\beta^{*} \leq-Q_{\min }^{*}\|e\|_{2}^{2}+\beta^{*} .
\end{aligned}
$$

Using (22), it follows that $\dot{V}_{\sigma} \leq-\gamma_{v} V_{\sigma}+\beta^{*}$. Note that the solutions of the differential equations $\dot{v}_{\sigma}(t)=$ $-\gamma_{v} v_{\sigma}(t)+\beta^{*}, v_{\sigma}(0)=v_{0} \geq 0$, are given by

$$
v_{\sigma}(t)=\frac{\beta^{*}}{\gamma_{v}}\left(1-e^{-\gamma_{v} t}\right)+v_{0} e^{-\gamma_{v} t} .
$$

Since the series of switching times $t_{1}+t_{2}+\ldots$ converges to infinity as time goes to infinity, the corresponding exponential converges to zero. It follows from the comparison principle [9] that $V_{\sigma(t)}(t) \leq v_{\sigma(t)}(t)$ when $V_{\sigma}(0) \leq v_{0}$. From (22) and (23) it is obtained that

$$
\|e\|_{2} \leq \frac{V_{\sigma}^{1 / 2}}{P_{\min }^{*} 1 / 2} \leq \frac{\left[\beta^{*}\left(1-e^{-\gamma_{v} t}\right) / \gamma_{v}+v_{0} e^{-\gamma_{v} t}\right]^{1 / 2}}{P_{\min }^{*}{ }^{1 / 2}} .
$$

Thus, when $t \rightarrow \infty$, the error converges to a neighborhood of the origin defined by (19). Therefore, theorem 2 is proven.

Remark 3 Note that (19) establishes a dependence between the parameter identification and the continuous state estimation error, since a large error in the parameter identification could yield a considerable error in the continuous state estimation.

\subsection{Observer Gain Design}

In this subsection the observer gain $L(\hat{\sigma})$ is synthesized, so that (18) is satisfied. By means of the Schur complement, (18) can be represented by the following LMIs

$$
\left[\begin{array}{cc}
-\frac{\varpi_{\sigma}}{6} I & P_{\sigma} \\
P_{\sigma} & \Xi_{i}^{\sigma}
\end{array}\right] \leq 0, \quad \forall \sigma=1, \ldots, q
$$


where $\Xi_{i}^{\sigma}=P_{\sigma} A_{i}^{\sigma}-M_{i}^{\sigma} C+A_{i}^{\sigma T} P_{\sigma}-C^{T} M_{i}^{\sigma}+\varpi_{\sigma} \delta_{A}^{2} I+$ $Q_{\sigma}$ and $M_{i}^{\sigma}=P_{\sigma} L(\hat{\sigma})$. The observer gain $L(\hat{\sigma})$ and the minimum value $\varpi_{\sigma}$ that satisfy (18) can be obtained by solving the minimization problem based on LMIs (24) subject to $P_{\sigma}=P_{\sigma}^{T}>0, Q_{\sigma}=Q_{\sigma}^{T}>0$ for all $\sigma=$ $1, \ldots, q$. Note that the corresponding value of $L(\hat{\sigma})$ will switch according to the discrete state estimation.

\section{SIMULATION RESULTS}

Consider the following LPV switched systems

$$
\begin{aligned}
& y^{(3)}(t)=-a_{0}(\sigma(t), \rho(t)) y(t)-a_{1}(\sigma(t), \rho(t)) \dot{y}(t) \\
& -a_{2}(\sigma(t), \rho(t)) \ddot{y}(t)+b_{0}(\sigma(t), \rho(t)) u(t)+\epsilon(t),
\end{aligned}
$$

with $u(t)=3(\sin (10 t)+\sin (t)+\sin (0.1 t)+\sin (0.01 t))$, $\rho(t)=\sin (0.1047 t)$, and $\epsilon(t)=\sum_{i=0}^{2} 0.2 y^{(i)}(t)+$ $b_{0}(\cdot) \nu_{1}(t)$ representing disturbances produced by parameter uncertainties and Gaussian noises $\nu_{1}(t)$, $\nu_{2}$, with zero mean and a variance of 0.003 to the input and output, respectively. The unknown varying parameters have the following structure: $a_{0}(1, \rho(t))=-5+2 \rho(t), a_{0}(2, \rho(t))=-11+2 \rho(t)$, $a_{1}(1, \rho(t))=-2+\rho(t), a_{1}(2, \rho(t))=-6+\rho(t)$, $a_{2}(1, \rho(t))=-9+3 \rho(t), a_{2}(2, \rho(t))=-17+3 \rho(t)$, $b_{0}(1, \rho(t))=4+\rho(t)$, and $b_{0}(1, \rho(t))=14+\rho(t)$. Therefore, the known parameter domains are given by $\Omega^{1}=[-7,-3] \times[-3,-1] \times[-12,-6] \times[3,5]$, and $\Omega^{2}=[-13,-9] \times[-7,-5] \times[-20,-14] \times[13,15]$. Simulations have been done in the MATLAB Simulink environment, with the Euler discretization method and sampling time $h=0.001[\mathrm{sec}]$. The discrete state $\sigma(t): \mathbb{R} \rightarrow \mathcal{Q}=\{1,2\}$ has the time evolution depicted in Fig. 6. The filter parameters are $\lambda_{1}=0.2222$, $\lambda_{2}=0.0247$ and $\lambda_{3}=0.0014$ and they provide a transient time $\xi=0.8889[\mathrm{sec}]$. The identification time window is $T=5$. In order to calculate the parameter identification error bound, the following information is thrown after some simulations, $\|f\|_{1}=3.6391,\|\Psi\|_{2}=27.2013$, $\phi^{+}=27.0659, \eta_{2}=272.8114, \epsilon^{+}=9.6000$ and $\epsilon_{\theta}=0.4055$. Hence, $\|\tilde{\theta}\|_{2} \leq 23.3948$ which provides $\delta=11.6974$. Then, the error parameter domains $\Omega_{\delta}^{1}$ and $\Omega_{\delta}^{2}$ are constructed, and Algorithm 1 is implemented. The simulations results are shown in Figs. 5 and 6 . The continuous state estimation is given by observer (16)-(17). Programming the solution of the minimization problem (24) on the LMI MatLab Toolbox with $\delta_{A}=3.1$ (calculated based on the known parameter domains and the parameter identification results given in Fig. 5), the following data are given: $\varpi_{1}=0.0703$, $\varpi_{2}=0.0374, L(1)=[161.1886,312.2134,-86.8298]^{T}$ and $L(2)=[115.1206,212.3914,-103.4344]^{T}$. Results are depicted by Figs. 7 and 8 .
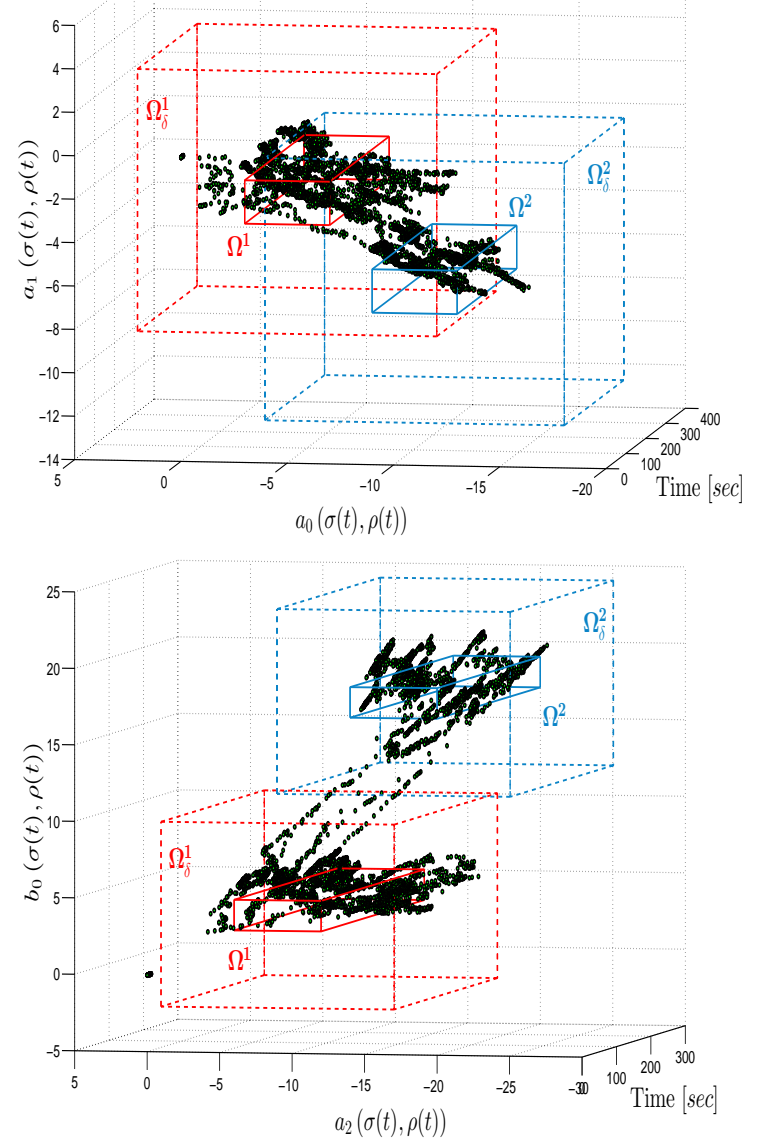

Fig. 5. Parameter Identification $\hat{\theta}_{L S}(t)$. The LSM (6) is simulated using the noisy input and noisy output information. In the above graphs the parameter estimation for the $L P V$ switched system at each time $k h$ is represented by a black point. It is easy to see how the points remain inside the corresponding set $\Omega_{\delta}^{\sigma(t)}$. Note that $\Omega_{\delta}^{1} \cap \Omega_{\delta}^{2} \neq \emptyset$. However, due to the fact that $\hat{\theta}_{L S}(\sigma(t), \rho(t)) \notin \Omega_{\delta}^{1} \cap \Omega_{\delta}^{2}$, a discrete state estimation is achieved.

\section{CONCLUSIONS}

In this paper the problem of discrete and continuous state estimation for a class of uncertain switched LPV systems is addressed. Parameter identification techniques are applied to realize an approximate identification of the switched LPV system parameters with certain uncertainties and/or disturbances. Making use of the parameter identification it is possible to achieve a discrete state estimation. Based on the discrete state estimation, a Luenberger-like hybrid observer is used for the continuous state estimation and synthesized based on LMIs. The simplicity of the proposed method is one of the main advantages of this paper. The workability of the proposed method is illustrated by simulations. 


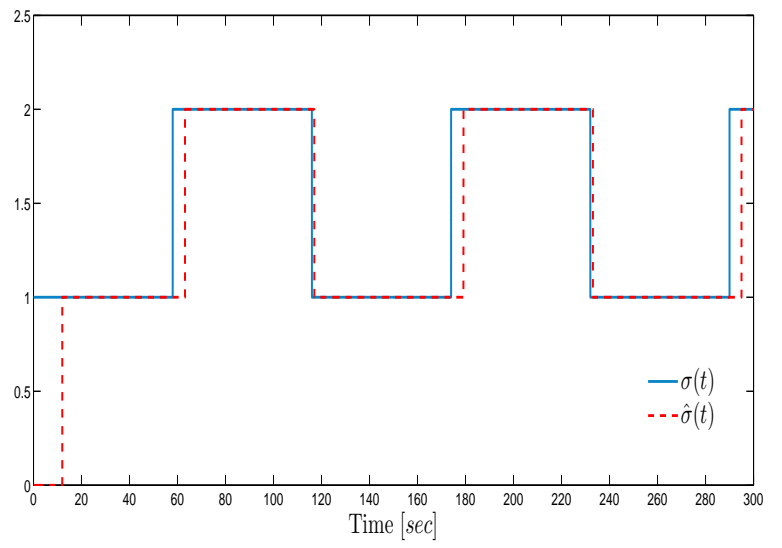

Fig. 6. Discrete State Estimation $\hat{\sigma}(k h)$. The discrete state estimation provided by Algorithm 1 is depicted in the top graph. It is worth mentioning that it is only possible to ensure the discrete state estimation after the time $T+\xi+\zeta=5.8899[\mathrm{sec}]$ after each switching time.

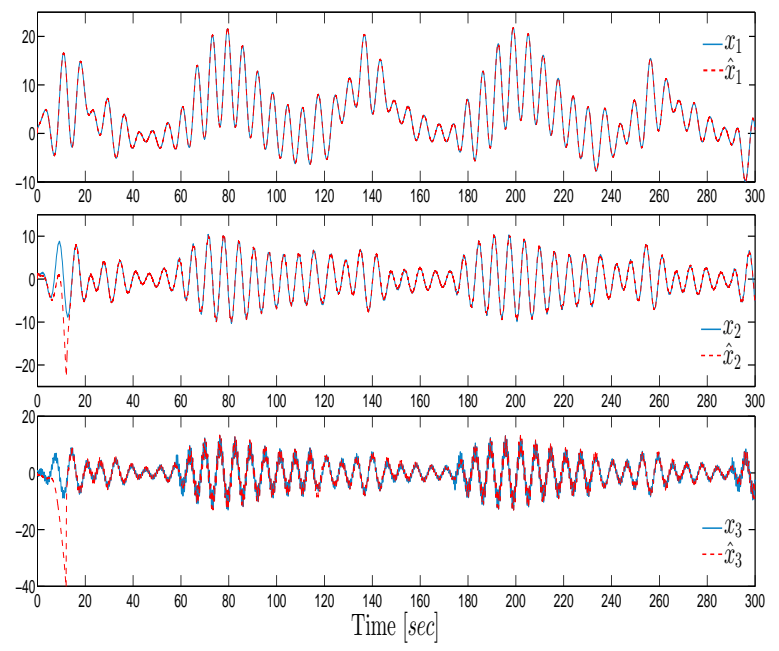

Fig. 7. Continuous State Estimation $\hat{x}(t)$.

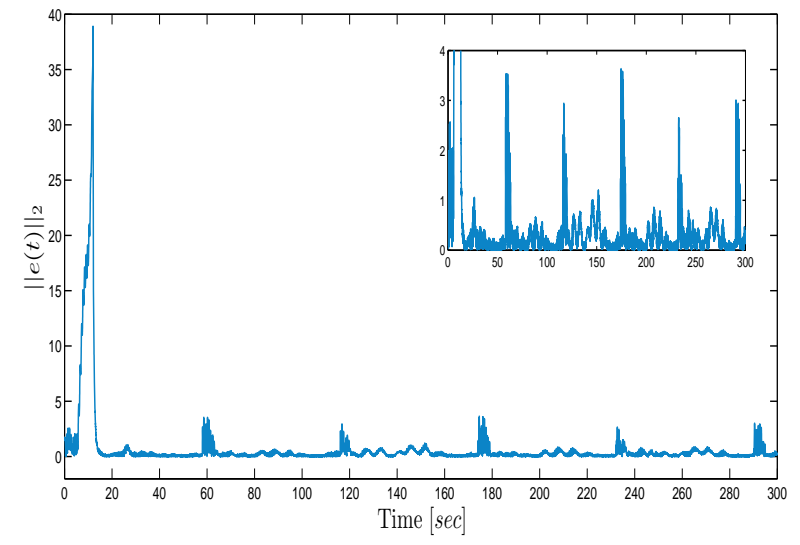

Fig. 8. Continuous State Estimation Error Norm $\|e(t)\|_{2}$.

\section{Acknowledgements}

The authors gratefully acknowledge the financial support from CONACyT 151855, 209247, CONACyT 209731 - CNRS 222629 Bilateral Cooperation Project Mexico-France, and IPN-SIP 20130983. This work was also supported in part by the Government of Russian Federation (Grant 074-U01) and the Ministry of Education and Science of Russian Federation (Project 14.Z50.31.0031).

\section{References}

[1] F. J. Bejarano and L. Fridman. State exact reconstruction for switched linear systems via a super-twisting algorithm. International Journal of Systems Science, 42(5):717-724, 2011.

[2] V. I. Bogachev. Measure Theory I. Springer-Verlag, Berlin, Heidelberg, 2007.

[3] M. Fliess, C. Join, and W. Perruquetti. Real-time estimation of the switching signal for perturbed switched linear systems. In Proceedings of the 3rd IFAC Conference on Analysis and Design of Hybrid Systems, pages 409-414, Zaragoza, Spain, 2009.

[4] T. Floquet, D. Mincarelli, A. Pisano, and E. Usai. Continuous and discrete state estimation in linear switched systems by sliding mode observers with residuals projection. In Proceedings of the 4th IFAC Conference on Analysis and Design of Hybrid Systems, pages 265-270, Eindhoven, The Netherlands, 2012.

[5] R.W. Hamming. Digital Filters. Dover books on engineering. Dover Publications, Mineola, NY, 2007.

[6] M. Hanifzadegan and R. Nagamune. Smooth switching LPV controller design for lpv systems. Automatica, 50:1481-1488, 2014.

[7] X. He, G.M. Dimirovski, and J. Zhao. Control of switched LPV systems using common Lyapunov function method and an F-16 aircraft application. In 2010 IEEE International Conference on Systems Man and Cybernetics, pages 386-392, Istanbul, Turkey, 2010.

[8] R Isermann and M. Münchhof. Identification of Dynamic Systems, An Introduction with Applications. Springer-Verlag, Berlin, 1st edition, 2011.

[9] H. Khalil. Nonlinear Systems. Prentice Hall, New Jersey, U.S.A., 2002.

[10] F. Lescher, J.-Y. Zhao, and P. Borne. Switching LPV controllers for a variable speed pitch regulated wind turbine. International Journal of Computers, Communications and Control, 1(4):73-84, 2006.

[11] D. Liberzon. Switching in Systems and Control. Systems and Control: Foundations and Applications. Birkhuser, Boston, MA, 2003.

[12] S. Lim and J.P. How. Modeling and $\mathcal{H}_{\infty}$ control for switched linear parameter-varying missile autopilot. IEEE Transactions on Control Systems Technology, 11(6):830-838, Nov 2003.

[13] L. Liu, X. Wei, and Z. Liu. Switching LPV control for the air path system of diesel engines. In Chinese Control and Decision Conference, pages 4167-4172, Shangai, P.R. China, 2008. 
[14] L. Ljung. System Identification: Theory for the User. Prentice Hall Information and System Sciences Series, New Jersey, second edition, 1999.

[15] B. Lu and F. Wu. Switching LPV control designs using multiple parameter-dependent Lyapunov functions. Automatica, 40:1973-1980, 2004.

[16] B. Lu, F. Wu, and S. W. Kim. Switching LPV control of an F-16 aircraft via controller state reset. IEEE Transactions on Control Systems Technology, 14(2):267-277, Mar 2006.

[17] J. Mohammadpour and C.W. Scherer. Control of Linear Parameter Varying Systems with Applications. Springer, New York, 2012.

[18] A.M. Nagy, G. Mourot, B. Marx, J. Ragot, and G. Schutz. Systematic multi-modeling methodology applied to an activated sludge reactor model. Industrial 83 Engineering Chemistry Research, 49(6):2790-2799, February 2010.

[19] N. Orani, A. Pisano, M. Franceschelli, A. Giua, and E. Usai. Robust reconstruction of the discrete state for a class of nonlinear uncertain switched systems. Nonlinear Analysis: Hybrid Systems, 5(2):220-232, 2011.

[20] B. Ramaswami and K. Ramar. Transformation of time-variable systems to canonical (phase-variable) form. IEEE Transactions on Automatic Control, 13(6):746-747, December 1968.

[21] H. Ríos, D. Efimov, J. Davila, T. Raïssi, L. Fridman, and A. Zolghadri. Non-minimum phase switched systems: Hosmbased fault detection and fault identification via volterra integral equation. International Journal of Adaptive Control and Signal Processing, 2013. DOI: 10.1002/acs.2448.

[22] H. Ríos, D. Mincarelli, D. Efimov, W. Perruquetti, and J. Davila. Discrete state estimation for switched LPV systems using parameter identification. In 2014 American Control Conference, pages 3243-3248, Portland, OR, USA, 2014.

[23] W.J. Rugh and J.S. Shamma. Research on gain scheduling. Automatica, 36:1401-1425, 2000.

[24] C.W. Scherer. Mixed $\mathcal{H}_{2} / \mathcal{H}_{\infty}$ control for time-varying and linear parametrically-varying systems. International Journal of Robust and Nonlinear Control, 6(9-10):929-952, 1996.

[25] J.S. Shamma. Analysis and design of gain scheduled control systems. Ph.D. Thesis, Department of Mechanical Engineering, MIT, 1988.

[26] L. Silverman. Transformation of time-variable systems to canonical (phase-variable) form. IEEE Transactions on Automatic Control, 11(2):300-303, April 1966.

[27] L. Vu and D. Liberzon. Invertibility of switched linear systems. Automatica, 44:949-958, 2008.

[28] L. Zhang, N. Cui, M. Liu, and Y. Zhao. Asynchronous filtering of discrete-time switched linear systems with average dwell time. IEEE Transactions on Circuits and Systems I, 58(5):1109-1118, 2011.

[29] L. Zhang and P. Shi. $\mathcal{L}_{2}-\mathcal{L}_{\infty}$ model reduction for switched LPV systems with average dwell time. IEEE Transactions on Automatic Control, 53(10):2443-2448, 2008.

[30] K. Zhou and J.C. Doyle. Essentials of robust control. Prentice-Hall, 1998. 